OnLine Journal of Biological Sciences 12 (1): 27-33, 2012

ISSN 1608-4217

(C) 2012 W. Phachonpai et al., This open access article is distributed under a Creative Commons Attribution

(CC-BY) 3.0 license

\title{
Effect of Dietary Kaempferia parviflora on Ischemic Brain Injury in the rat
}

\author{
${ }^{1}$ Wathita Phachonpai, ${ }^{2}$ Sinthuporn Maharun, \\ ${ }^{3}$ Supaporn Muchimapura, ${ }^{3}$ Jintanaporn Wattanathorn and ${ }^{3}$ Terdthai Tong-Un \\ ${ }^{1}$ Division of Physiology, School of Medical Science, \\ University of Phayao, Phayao, 56000, Thailand \\ ${ }^{2}$ Division of Allied Health Science, School of Physical Therapy, \\ University of Phayao, Phayao, 56000, Thailand \\ ${ }^{3}$ Department of Physiology, Faculty of Medicine, \\ Khon Kaen University, Khon Kaen, 40002, Thailand
}

\begin{abstract}
Problem statement: Stroke often causes irreversible neuronal damage and related behavioral deficits. The lack of effective and widely applicable pharmacological treatments for ischemic stroke patients may explain a growing interest in traditional plant medicines. We investigated the ability of consuming Kaempferia parviflora extract, a Thai medicinal plant reputed for neuroprotective effects against brain damage, reduced infarct volume and improve neurological outcome in rats with permanent right Middle Cerebral Artery Occlusion (MCAO). Approach: Male adult Wistar rats were administrated an alcoholic extract of KP orally once daily at a period of 14 days before and 7 days after MCAO. Neurological function assessment was performed at 7 days after MCAO using the 6-points postural reflex test. The brain infarct volume and the densities of survival neurons in hippocampus were also determined at the end of experiment. Results: Oral administration with $\mathrm{KP}$ at a dose of $200 \mathrm{mg} \mathrm{kg}^{-1} \mathrm{BW}$ significantly improved the neurological behavior performances and reduced the infarct volume when compared to the vehicle treated group. The reductions of survival neurons densities were also mitigated. Conclusion: The consumption of KP extract may have the possibility of protective effect against neurological disorders such as brain ischemia, while further investigations about precise underlying the defense mechanism against cerebral ischemia are still required.
\end{abstract}

Key words: Kaempferia parviflora, neuroprotective, traditional plant medicine, cerebral ischemia, neurological behavior deficit, Middle Cerebral Artery Occlusion (MCAO)

\section{INTRODUCTION}

Ischemic stroke is a leading cause of death and severe disability and place a large burden on healthcare and social service resources in most countries (Plaisier et al., 2008). Therapeutic strategies to limit infarct size and improve functional outcome after stroke injury are aimed at rescuing this potential reversible ischemic region (Wang et al., 2008). To date, the available efficacies of drugs treatment for stroke condition are still very limited due to their adverse side effects.

Accumulating lines of evidence reported that oxidative stresses such as generation of damaging reactive oxygen species will lead to cell death under ischemic condition (Gilgun-Sherki et al., 2002). Furthermore, oxidative stress involves redox signaling to molecular mediators of inflammation pathways, which induce further cell damage (Chang et al., 2005; Ritz et al., 2008). Increasing evidence has indicated that ischemia, which occurs due to blockage of the Middle Cerebral Artery or due to recirculation of blood flow, causes oxidative stress that may potentiate ischemic injury (Jittiwat et al., 2009). In recent times, growing evidences support participation of phenolic antioxidants from traditional plant medicines to posses as neuroprotective agents against cerebral ischemia (Rice et al., 1981; Calapai et al., 2000; Phachonpai et al., 2010). 
Traditional plant medicines are useful alternative source of novel drugs containing various pharmacological activities. Zingiberaceae, the Ginger family, includes many important medicinal plants and is a rich source of biologically active compounds (Ojewole, 2006; Stoilova et al., 2007). In Thailand, a member of the Zingiberaceae family, Kaempferia parviflora (Krachaidum; KP), is used as a folk medicine. The rhizomes of KP are applied as a medicine for a treatment of hypertension (Tewtrakul et al., 2008), anti-male impotence (Sudwan et al., 2006) and anti-gastric ulcer activity (Rujjanawate et al., 2005). Furthermore, the extracts and flavone derivatives from the rhizome of KP could exhibit antiinflammatory and antioxidant activities (Tewtrakul et al., 2009; Sakong et al., 2011). Moreover, administration of KP extract has been proven to reduce the brain damage, improve memory impairment (Spencer, 2009) and induce vasorelaxation activity (Tep-Areenan et al., 2010).

Based on the flavonoid ingredients, antioxidant activity and biological effects of KP rhizome extract, the present study therefore sought to confirm the usefulness of KP extract against the neuronal damage, brain infarct and neurological deficits in rats with permanent right Middle Cerebral Artery Occlusion (MCAO) model.

\section{MATERIALS AND METHODS}

Plant material and preparation of the crude extract: Kaempferia parviflora rhizomes (Rom gloa variation) were collected from tombon boh-park, charttrakarn, Phitsanulok, Thailand. The herbarium was authenticated by associate professor bungorn sripanidkulchai and deposited as voucher specimen (KP-CRD 10D) at center for research and development of herbal health product, faculty of pharmaceutical sciences, khon kaen university, Thailand. The dried plant rhizome powder was macerated in $95 \%$ ethanol for 4 days $\left(1 \mathrm{~kg} \mathrm{~L}^{-1}\right)$ with occasionally stirring. After filtration, the residual was further repeat macerated with 95\% ethanol and then the filtrate were combined and dried by freeze dryer. The percent yield of the final product was $4.82 \%$. The extract contained total flavonoids content approximate $40.37 \mathrm{mg} \mathrm{g}^{-1}$ dried powder consisting 2 main constituents 5, 7dimethoxyflavone $\left(8.789 \mathrm{mg} \mathrm{g}^{-1}\right)$ and $3,5,7,3^{\prime}, 4^{\prime}-$ pentamethoxyflavone $\left(9.858 \mathrm{mg} \mathrm{g}^{-1}\right)$.

Animals: Healthy male Wistar rats weighing 300-350 gm and aging 8 weeks were obtained from National
Laboratory Animal Center, Salaya, Nakorn Pathom. They were housed in group of 5 per cage in standard metal cages at $2 \pm 22^{\circ} \mathrm{C}$ on $12: 12 \mathrm{~h}$ light-dark cycle. All animals were given access to food and water ad libitum. Experiments were performed to minimize animal suffering in accordance with the internationally accepted principles for laboratory use and care of European Community (EEC directive of 1986; 86/609/EEC) and approved by the Ethical Committee of the Khon Kaen University.

Experimental protocol: All rats were randomly assigned to 6 groups ( $\mathrm{n}=8$ in each group). Group I: Naïve intact control rats. Group II: sham-operated group. Group III: Vehicle (2\% SCMC), which used as vehicle to a desired concentration. Group IV-VI: Rats were treated with different doses of the alcoholic extract of KP $(100,200$ and $300 \mathrm{mg} \mathrm{kg}$ $\left.{ }^{-1} \mathrm{BW}\right)$, respectively. The doses of KP were selected on the basis of previous studies conducted in laboratory and those reported in literature.

All rats were orally assigned substances via the intragastric feeding tube once daily for 14 days before and 7 days after MCAO. Neurological evaluation was determined at 7th day after MCAO. Later on, the dosage of KP that produced the improvement of neurological score was selected for further evaluation the infarct volume and the densities of survival neurons in hippocampus.

Focal cerebral ischemia induction: Anesthesia was induced with intraperitoneal injection of thiopental sodium at dose of $50 \mathrm{mg} \mathrm{kg}{ }^{-1} \mathrm{BW}$. Focal cerebral ischemia was performed according to modified method of Longa et al. (1989). Briefly, the right common carotid artery and the right external carotid artery were exposed through a ventral midline neck incision and were ligated proximally. A silicone coated nylon monofilament (4-0) suture (USS DG ${ }^{\mathrm{TM}}$ sutures; Tyco Healthcare group LP, Connecticut, USA) with its tip rounded by heating near a flame was inserted through an arteriectomy in the common carotid artery just below the carotid bifurcation, and then advanced into the internal carotid artery approximately $17-18 \mathrm{~mm}$ distal to the carotid bifurcation until a mild resistance was felt. Occlusion of the origins of the anterior cerebral artery, the middle cerebral artery, and the posterior communicating artery was thereby achieved. Then, the wound was sutured, the rats were returned to their cages with free access to food and water. The incision sites were infiltrated with $10 \%$ povidoneiodine solution for anti-septic postoperative care. 
Assessment of neurological deficit: All animals were subjected to neurological evaluation by using the 6-points postural reflex test according to the method of Bederson et al. (1986) and SchmidElsaesser et al. (1998). The deficit was graded from 0-5 as follow: Grade 0: No spontaneous activity; Grade 1: Spontaneous circling; Grade 2: Circling if pulled by tail; Grade 3: Lowered resistance to lateral push without circling; Grade 4: Contra lateral forelimb flexion; Grade 5: No apparent deficit.

Determination of infarct volume: The infarct volume was assessed with 2\% 2, 3, 5-Triphenyl Tetrazolium Chloride (TTC) solution in saline for $30 \mathrm{~min}$ at $37^{\circ} \mathrm{C}$ and photographed. These images were analyzed using a computerized image analysis system (Optimas 6.1; Media Cybernetics, Silver Springs, MD, USA) to measure the infarct area (Shimamura et al., 2006). The infarct area in each slice was calculated by subtracting the normal ipsilateral area from that of the contralateral hemisphere to reduce errors due to cerebral edema and was presented as a percentage relative to the area of the contralateral hemisphere.

Histological procedure: As noted above, after behavioral tests, rats were killed. The brains were dissected out and placed in $4 \%$ paraformaldehyde solution as fixative. Histopathological sections were made using Nissl staining to identify the survival neurons in hippocampus. Five coronal sections of each rat in each group were studied quantitatively. Neuronal counts in hippocampus were performed by eye using a 40x objective with final field $225 \mu^{2}$ according to the following stereotaxic coordinates: AP-4.8 mm, lateral $\pm 2.4-6 \mathrm{~mm}$ and depth 3-8 $\mathrm{mm}$. The observer was blinded to the treatment at the time of analysis. Viable stained neurons were identified on the basis of a stained soma with at least two visible processes. Counts were made in five adjacent fields and the mean number extrapolated to give the total number of neurons per $225 \mu \mathrm{m}^{2}$. All data are represented as number of neurons per $225 \mu^{2}$

Statistical analysis: All analyses were performed using SPSS for Windows version 13 software (SPSS Inc., Chicago, IL, USA). Results are expressed as the mean \pm S.E.M. Statistical differences among the experimental groups were tested using one way Analysis of Variance (ANOVA), followed by Dunnett's t-test. The p-value < 0.05 was considered statistically significant.

\section{RESULTS}

Improvement of Kaempferia parviflora on neurological function: There was no neurobehavioral dysfunction symptom in rats of the sham-operation group.

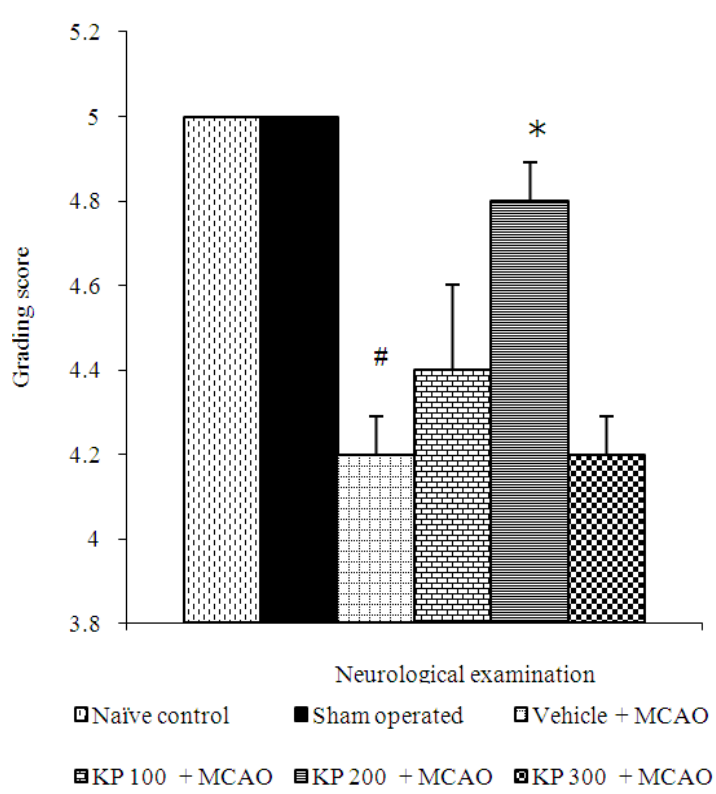

Fig. 1: Effect of KP extracts at dose of 100, 200 and $300 \mathrm{mg} \mathrm{kg}^{-1} \mathrm{BW}$ on the neurological score deficits. Values given are the mean \pm S.E.M. $(\mathrm{n}=8)$. \# $\mathrm{p}<0.05$ as compared to sham operation treated group, $* \mathrm{p}<0.05$ as compared to vehicle treated group

The Bederson's scores in the vehicle treated were lower than that in the sham-operation group ( $\mathrm{p}$-value <0.05). Interestingly, treatment with KP at a dose of $200 \mathrm{mg}$ $\mathrm{kg}^{-1} \mathrm{BW}$, the neurological deficit score was significantly improved compared with vehicle treated group (p-value $<0.05$ ) while the low and high doses of KP extract did not produce the significant changes on this parameter (Fig. 1).

Effect of Kaempferia parviflora reduces the volume of infarction: The infarct volume was significantly higher in the vehicle treated group than in .... shamoperation group (p-value $<0.05$ ) while treatment with $\mathrm{KP}$ at a dose of $200 \mathrm{mg} \mathrm{kg}^{-1} \mathrm{BW}$ could reduce the brain infarct volume only in the cortical whereas no significant changes in subcortical areas as compared with vehicle treated group (p-value $<0.05$; Fig. 2).

Neuroprotective effect of Kaempferia parviflora: The Nissl-stained slices of rat's brain showed intense, rich Nissl bodies in the sham-operated group whereas the vehicle treated group, Nissl bodies were significantly reduced in all areas of the hippocampus. Notably, KP at a dose of $200 \mathrm{mg} \mathrm{kg}^{-1} \mathrm{BW}$ could mitigate the reduction of Nissl bodies after MCAO in all areas of the hippocampus ( $\mathrm{p}<0.05$ all; Fig. 3). 


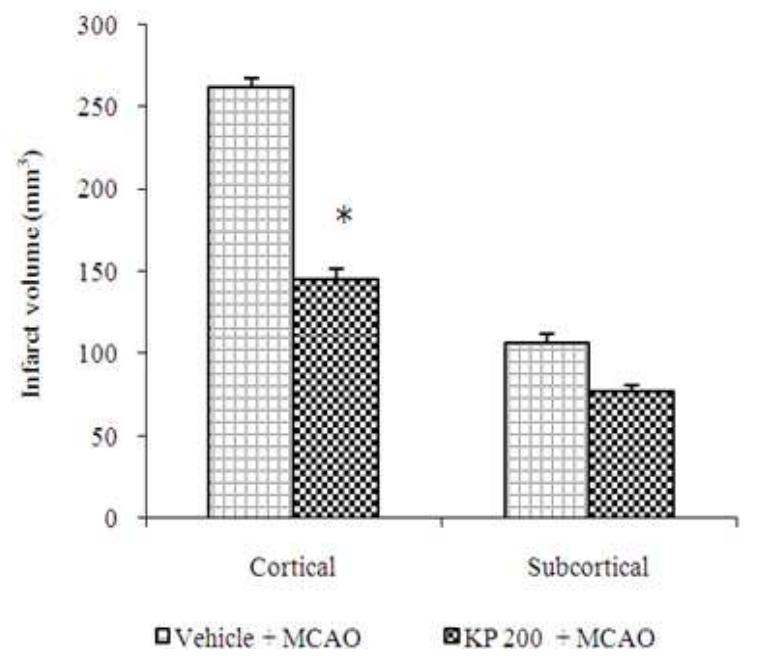

Fig. 2:Effect of KP extract at a dose of $200 \mathrm{mg} \mathrm{kg}^{-1}$ $\mathrm{BW}$ on the infarct volume. Values given are the mean \pm S.E.M. $(\mathrm{n}=8)$. ${ }^{*} \mathrm{p}<0.05$ as compared to vehicle treated group



Fig. 3: Effect of KP extract at a dose of $200 \mathrm{mg}$ $\mathrm{kg}^{-1} \mathrm{BW}$ on the densities of survival neurons in hippocampus. Values given are the mean \pm S.E.M. $(\mathrm{n}=8)$ and $\mathrm{p}<0.05$ as compared to naïve control group, \# $\mathrm{p}<0.05$ as compared to sham operation treated group, ${ }^{*} \mathrm{p}<0.05$ as compared to vehicle treated group

\section{DISCUSSION}

Despite the impacts of stroke on several million people worldwide every year, no treatment exists to minimize the neurological damage. People who suffer stroke often experience persistent functional impairment. Accumulating lines of data reported that dietary enrichment with nutritional antioxidants could reduce brain damage and improve behavior functional deficits (Suk et al., 2002; Koo et al., 2004). Evidence suggests that antioxidant activities of plants used in traditional herbal medicine have been shown to be neuroprotective when given before or shortly after induction of focal cerebral ischemia (Hall et al., 1988; Liu et al., 1989). The results of the present study revealed that KP oral administration significantly ameliorated the neurological deficit, reduced the infarct volume and mitigated the degeneration of survival neurons in hippocampus within 7 days postischemia. These encouraging results may have future clinical importance because of the increase using of herbal supplements by the general population. However, this study did not investigate about the precise underlying the defense mechanism of KP extract could protect against cerebral ischemia; this is planned in future studies.

Middle Cerebral Artery Occlusion (MCAO) is most commonly model used to induce experimental focal cerebral ischemia (Garcia et al., 1995). The advantages of the MCAO model are its reproducibility and ease of reperfusion. Besides, the type of ischemic injury observed is similar to that found in human (Hacke et al., 1996). Therefore, MCAO model has been employed in the present study.

The brain injury induced by MCAO leads to neurobehavioral impairment. We performed the modified by using 6-points postural reflex test to measure neurobehavioral changes. In this study, all rats in the operation (MCAO) groups showed a neurologic deficit after MCAO injury. Surprisingly, at 7 days after MCAO, the rats treated with KP at a dose of $200 \mathrm{mg}$ $\mathrm{kg}^{-1} \mathrm{BW}$ were improved the neurological deficit scores compared to the vehicle treated group. Unfortunately, the low and high doses of KP extract did not produce the significant changes on this parameter. One possible explanation for this phenomenon might be due the KP extract at a low dose might possibly fail to raise the concentration of active ingredient in the extract to the therapeutic level. On the other hand, the increasing a dose of the crude extract of KP might also increase the concentration of some ingredients which masked the effect of the active ingredient. These findings are consistent with in vivo observations as well as previous studies (Hawiset et al., 2011). However, the animal model to induce the lesion, the dose of KP extract and the duration of treatment of the plant extract were different. 
Furthermore, the permanent MCAO in rat model cause cerebral cell death, resulting in local infarction. The infarct area represents the degree of brain injury (Ishibashi et al., 2004). In the present study, rats were oral administered with KP extract at a dose of $200 \mathrm{mg}$ $\mathrm{kg}^{-1} \mathrm{BW}$ for 14 days before and 7 days after MCAO showed markedly the reduction in infarction volume only in the cortex whereas no significant change in subcortical versus vehicle administered rats. As is the case in clinical stroke, infarct volume does not necessarily correlate with functional outcome in patients (Gladstone et al., 2002). This observation needs further investigation in near future.

Previous study had demonstrated that the behavior impairments in stroke patients were associated with the severity of brain infarction, which in turn depended on the densities of survival neurons in the cortex, the hippocampus and the striatum (Leist and Jaattela, 2001). Our results clearly demonstrated that the KP extract at a dose of $200 \mathrm{mg} \mathrm{kg}{ }^{-1} \mathrm{BW}$ significantly enhanced neuron densities in all areas as mention. Notably, KP extract could increase the densities of neurons in CA1 more than those in naive intact control. Therefore, the increased neuron densities in CA1might involve its neurotrophic action. However, further researches particularly BrdU staining are needed to identify the active constituent of the KP extract responsible for its neurotrophic activity.

Recent studies have demonstrated that Nitric Oxide (NO) levels are associated directly with the development of brain injury in strokes and other neuropathological disorders in humans (Veltkamp et al., 2002). NO can be neuroprotective or neurotoxic during cerebral ischemia, depending on the Nitric Oxide Synthase (NOS) isoform involved. eNOS produces NO with beneficial effects (vasodilation), whereas NO overproduction by nNOS or iNOS during ischemia is cytotoxic (Li et al., 2002). Numerous studies of KP rhizomes extract have reported that they are the effective upregulate the expression of eNOS, iNOS express inhibition, free radical scavenger and antioxidant (Wattanapitayakul et al., 2007; Sae-Wong et al., 2011). We believe that the effects observed in our study may be mediated through one of these KP mechanisms.

To the best of our knowledge, this is the first report to show the neuroprotective effects of KP oral administration against focal cerebral ischemia. These results suggested that KP extract holds promise as a neuroprotectant agent for use as a food supplement or functional food to reduce ischemia injury. Further studies are warranted to pursue the interesting lead emerging from the present results to exploit the full therapeutic potential of KP in ischemic stroke.

\section{CONCLUSION}

In conclusion, we have shown that the KP supplementation significantly prevents the development of ischemic injury in stroke condition. Recently, selfmedication with dietary supplements has become increasingly popular for health-maintenance and prevention of life-style related diseases including cerebral ischemia. Our results may help the development of preventive medicine against the cerebrovascular diseases.

\section{ACKNOWLEDGMENT}

This study was supported by the National Research Council of Thailand and Integrative Complimentary Alternative Medicine Research and Development Group, Khon Kaen University, Thailand. The authors have no conflict of interest to report.

\section{REFERENCES}

Bederson, J.B., L.H. Pitts, M. Tsuji, M.C. Nishimura, R.L. Davis and H. Bartkowski, 1986. Rat middle cerebral artery occlusion: Evaluation of the model and development of a neurologic examination. Stroke, 17: 472-476. DOI: 10.1161/ 01.STR.17.3.472

Calapai, G., A. Crupi, F. Firenzuoli, M.C. Marciano M, F. Squadrito, G. Inferrera et al., 2000. Neuroprotective effects of Ginkgo biloba extract in brain ischemia are mediated by inhibition of nitric oxide synthesis. Life Sci., 67: 2673-2683. DOI: 10.1016/S0024-3205(00)00858-4.

Chang, C.Y., J.Y. Chen, D. Ke and M.L. Hu, 2005. Plasma levels of lipophilic antioxidant vitamins in acute ischemic stroke patients: Correlation to inflammation markers and neurological deficits. Nutrition, 21: $\quad 987-993 . \quad$ DOI: 10.1016/j.nut.2005.02.010.

Garcia, J.H., S. Wagner, K.F. Liu and X.J. Hu, 1995. Neurological deficit and extent of neuronal necrosis attributable to middle cerebral artery occlusion in rats. Stroke, 26: 627-663. DOI: 10.1161/01.STR.26.4.627.

Gilgun-Sherki, Y., Z. Rosenbuam, E. Melamed and D. Offen D, 2002. Antioxidant therapy in acute central nervous injury: Current state. Pharmacol. Rev., 54: 271-284. DOI: $10.1124 /$ pr.54.2.271

Gladstone, D.J., S.E. Black and A.M. Hakim, 2002. Toward wisdom from failure: Lessons from neuroprotective stroke trials and new therapeutic directions. Stroke, 33: 2123-2136. DOI: 10.1161/01.STR.0000025518.34157.51 
Hacke, W., S. Schwab, M. Horn, M. Spranger, M.D. Georgia and R.V. Kummer, 1996. 'Malignant' middle cerebral artery territory infarction: Clinical course and prognostic signs. Arch. Neurol., 53: 309-315.

DOI: 10.1001/archneur.1996.00550040037012

Hall, E.D., K.E. Pazara and J.M. Braughler, 1988. 21Aminosteroid lipid peroxidation inhibitor U74006F protects against cerebral ischemia in gerbils. Stroke, 19: 997-1002. DOI: 10.1161/01.STR.19.8.997.

Hawiset, T., S. Muchimapura, J Wattanathorn and B Sripanidkulchai, 2011. Screening neuropharmacological activities of kaempferia parviflora (krachai dam) in healthy adult male rats. Am. J. Applied Sci., 8: 695-702. DOI: 10.3844/ajassp.2011.695.702

Ishibashi, S., T. Kuroiwa, N. Katsumata, S.L. Yuan and S. Endo et al., 2004. Extrapyramidal motor symptoms versus striatal infarction volume after focal ischemia in mongolian gerbils. Neuroscience, 127: 269-275.

DOI: 10.1016/j.neuroscience.2004.04.039

Jittiwat, J., J. Wattanathorn, T. Tongun, S. Muchimapura and C. Bunchonglikitkul, 2009. Porcine brain extract attenuates memory impairments induced by focal cerebral ischemia. Am. J. Applied Sci., 6: 1662-1668. DOI: 10.3844/ajassp.2009.1662.1668

Koo, B.S., W.C. Lee, Y.C. Chang and C.H. Kim, 2004. Protective effects of alpinae oxyphyllae fructus (Alpinia oxyphylla MIQ) water-extracts on neurons from ischemic damage and neuronal cell toxicity. Phytother Res., 18: 142-148. DOI: $10.1002 /$ ptr. 1382

Leist, M. and M.M. Jaattela, 2001. Four deaths and a funeral: From caspases to alternative mechanisms. Nat. Rev. Mol. Cell Biol., 2: 589-598. DOI: 10.1038/35085008

Li, L., Y.M. Shen, X.S. Yang, W. L. Wu and B.G. Wang et al., 2002. Effects of spiramine $\mathrm{T}$ on antioxidant enzymatic activities and nitric oxide production in cerebral ischemia-reperfusion gerbils. Brain Res., 944: 205-209. DOI: 10.1016/S0006-8993(02)02892-5

Liu, T.H., J.S. Beckman, B.A. Freeman, E.L. Hogan and C.Y. Hsu, 1989. Polyethylene glycolconjugated superoxide dismutase and catalase reduce ischemic brain injury. Am. J. Physiol., 256: H589-H593. PMID: 2492771

Longa, E.Z., P.R. Weinstein, S. Carlson and R. Cummins, 1989. Reversible middle cerebral artery occlusion without craniectomy in rats. Stroke., 20: 84-91. DOI: 10.1161/01.STR.20.1.84
Ojewole, J.A.O., 2006. Analgesic, antiinflammatory and hypoglycaemic effects of ethanol extract of Zingiber officinale (Roscoe) rhizomes (Zingiberaceae) in mice and rats. Phytother. Res., 20: 764-772. DOI: 10.1002/ptr.1952

Phachonpai, W., J. Wattanathorn, S. Muchimapura, T. Tong-Un and D. Preechagoon, 2010. Neuroprotective effect of Quercetin encapsulated liposomes: A novel therapeutic strategy against Alzheimer's disease. Am. J. Applied Sci., 4: 480485. DOI: 10.3844/ajassp.2010.480.485

Plaisier, F., M. Bastide, T. Ouk, O. Pétrault, M. Laprais, S. Stolc, et al., 2008. Stobadine-induced hastening of sensorimotor recovery after focal ischemia/reperfusion is associated with cerebrovascular protection. Brain Res., 1208: 240249. DOI: 10.1016/j.brainres.2008.02.007

Rice, J.E., R.C. Vannucci and J.B. Brierley, 1981. The influence of immaturity on hypoxic-ischemic brain damage in the rat. Annal. Neurol., 92: 131-141. DOI: 10.1002/ana.410090206

Ritz, M.F., P. Ratajczak, Y. Curin, E. Cam, A. Mendelowitsch, F. Pinet et al., 2008. Chronic treatment with red wine polyphenol compounds mediates neuroprotection in a rat model of ischemic cerebral stroke. J. Nutr., 138: 519-525. PMID: 18287360

Rujjanawate, C., D. Kanjanapothi, D. Amornlerdpison and S. Pojanagaroon, 2005. Anti-gastric ulcer effect of Kaempferia parviflora. J. Ethnopharmacol., 102: 120-122. DOI: 10.1016/j.jep.2005.03.035

Sae-Wong, C., H. Matsuda, S. Tewtrakul, P. Tansakul, S. Nakamura, Y. Nomura et al., 2011. Suppressive effects of methoxyflavonoids isolated from Kaempferia parviflora on inducible Nitric Oxide Synthase (iNOS) expression in RAW 264.7 cells. J. Ethnopharmacol., 136: 488-495. DOI: 10.1016/j.jep.2011.01.013

Sakong, P., T. Khampitak, U. Cha'on, C. Pinitsoontorn, P. Sriboonlue, P. Yongvanit et al., 2011. Antioxidant activity and bioactive phytochemical contents of traditional medicinal plants in northeast Thailand. J. MPR., 5: 6822-6831. DOI: 10.5897/JMPR11.1222

Schmid-Elsaesser, R., S. Zausinger, E. Hungerhuber, A. Baethmann and H.J. Reulen, 1998. A critical reevaluation of the intraluminal thread model of focal cerebral ischemia: Evidence of inadvertent premature reperfusion and subarachnoid hemorrhage in rats by laser-Doppler flowmetry. Stroke, 29: 2162-2170. DOI: 10.1161/01.STR.29.10.2162 
Shimamura, N., G. Matchett, T. Tsubokawa, H. Ohkuma and J. Zhang, 2006. Comparison of silicon-coated nylon suture to plain nylon suture in the rat middle cerebral artery occlusion model. J. Neurosci. Methods, 156: 161-165. DOI: 10.1016/j.jneumeth.2006.02.017

Spencer, J.P., 2009. Flavonoids and brain health: Multiple effects underpinned by common mechanisms. Genes Nutr., 4: 243-250. DOI: 10.1007/s12263-009-0136-3

Stoilova, I., A. Krastanov, A. Stoyanova, P. Denev and S. Gargova, 2007. Antioxidant activity of a ginger extract (Zingiber officinale). Food Chem., 102: 764-770. DOI: 10.1016/j.foodchem.2006.06.023

Sudwan, P., K. Saenphet, S. Saenphet and S. Suwansirikul, 2006. Effect of kaempferia parviflora wall. ex. Baker on sexual activity of male rats and its toxicity. Southeast Asian J. Trop. Med. Public Health., 37: 210-215. PMID: 17547083

Suk, K., S.Y. Kim, K. Leem, Y.O. Kim and S.Y. Park et al., 2002. Neuroprotection by methanol extract of uncaria rhynchophylla against global cerebral ischemia in rats. Life Sci., 70: 2467-2480. PMID: 12173411

Tep-Areenan, P., K. Ingkaninan and D.M. Randall, 2010. Mechanisms of Kaempferia Parviflora Extract (KPE)- induced vasorelaxation in the rat aorta. Asian Biomed., 4: 103-111.
Tewtrakul, S., S. Subhadhirasakul and S. Kummee, 2008. Anti-allergic activity of compounds from Kaempferia parviflora. J. Ethnopharmacol., 116: 191-193. PMID: 18077118

Tewtrakul, S., S. Subhadhirasakul, C. Karalai, C. Ponglimanont and S. Cheenpracha, 2009. Antiinflammatory effects of compounds from Kaempferia parviflora and boesenbergia pandurata. Food Chem., 115: 534-538. DOI: 10.1016/j.foodchem.2008.12.057

Veltkamp, R., N. Rajapakse, G. Robins, M. Puskar, K. Shimizu and D. Busija, 2002. Transient focal ischemia increases endothelial nitric oxide synthase in cerebral blood vessels. Stroke, 33: 2704-2710. DOI: 10.1161/01.STR.0000033132.85123.6A

Wang, C., J.L. Liu, H.F. Sang, Y. Lu and H.L. Dong et $a l .$, 2008. Therapeutic time window of flurbiprofen axetil's neuroprotective effect in a rat model of transient focal cerebral ischemia. Chin. Med. J., 121: 2572-2577. PMID: 19187598

Wattanapitayakul, S.K., M. Suwatronnakorn, L. Chularojmontri, A. Herunsalee, S. Niumsakul, S. Charuchongkolwongse et al., 2007. Kaempferia parviflora ethanolic extract promoted nitric oxide production in human umbilical vein endothelial cells. J. Ethnopharmacol., 110: 559-62. DOI: 10.1016/j.jep.2006.09.037 